\title{
Comparaison de la qualité des œufs et des performances de croissance des poussins locaux des zones urbaines et rurales de l'Ouest-Cameroun
}

\author{
T. C. KEAMBOU ${ }^{1 *}$, B. BOUKILA ${ }^{2}$, G. MOUSSOUNDA ${ }^{2}$ et Y. MANJELI $^{1}$ \\ ${ }^{I}$ Faculté d'Agronomie et des Sciences Agricoles, Université de Dschang-Cameroun. \\ ${ }^{2}$ Institut National Supérieur d'Agronomie et de Biotechnologies, Université des Sciences et \\ Techniques de Masuku-Gabon. \\ *Auteur correspondant, E-mail : christike2002@yahoo.fr; B.P. 222 Dschang-Cameroun.
}

\section{RESUME}

Dans le but de décrire la qualité des œufs et d'évaluer les performances de croissance des poussins locaux des zones urbaines et rurales de l'Ouest-Cameroun, il a été procédé à la collecte de 640 œufs de poule locale équitablement répartis entre trois localités urbaines et trois localités rurales de l'Ouest-Cameroun. Ces œufs ont été d'abord morphologiquement caractérisés, puis incubés artificiellement pour produire des poussins qui ont servi à l'étude des performances de croissance des poussins issus des zones urbaines et rurales. Il en ressort que les mensurations des œufs ont été comparables $(\mathrm{P}>0,05)$ entre les zones rurales et urbaines: en majorité de forme ovale ( 86,9 et $95 \%$ ), de couleur brune ( 86,25 et $83 \%$ ), d'un poids moyen variant de 44 à $46 \mathrm{~g}$ et d'indice environ 73. La fertilité des œufs a été plus élevée $(\mathrm{P} \leq 0,05)$ en zone rurale $(64,2 \%)$ contre $(43,5 \%)$ en zone urbaine, mais avec des taux d'éclosion comparables (62,3 et 59,7\%). Les performances de croissance ont été comparables $(\mathrm{P}>0,05)$ entre les deux zones, malgré la supériorité apparente des poussins de la zone urbaine pour la plupart des paramètres étudiés: poids à l'éclosion ( 28 et 30 g), poids à 5 semaines (255 et 273 g), consommation hebdomadaire (199 et $203 \mathrm{~g}$ ), gain moyen quotidien (7,18 et 7,82 g). Bien que la variabilité soit faible entre les deux zones pour la qualité des œufs et les performances de croissance, l'étude a révélé une légère supériorité des performances de croissance de la poule en zone urbaine, ce qui à l'échelle d'une production industrielle ne serait pas négligeable.

(C) 2009 International Formulae Group. All rights reserved.

Mots clés : productivité, poule locale, Cameroun

\section{INTRODUCTION}

Le secteur agricole joue un rôle important dans l'économie camerounaise. Le sous secteur élevage représente $16 \%$ de la production agricole et constitue une source de revenu pour environ 30\% de la population (INS-Cameroun, 2001). De par ses avantages (Tchoumboué et al., 2000), l'aviculture constitue un moyen rapide d'accroître la production de viande. Elle joue d'importantes fonctions socio-économique et socioculturelle. Les effectifs nationaux se situent à plus de 35 millions répartis dans les systèmes intensif et extensif (Fotsa et al., 2007).
Le système extensif, considéré traditionnel ou villageois, est dominé par la poule de race locale qui rassemble plus de $80 \%$ des effectifs totaux du pays (Tchoumboué et al., 2000). Ce système continuera de jouer un rôle très important comme source de protéine et de revenu pour le milieu rural où vit près de $80 \%$ de la population (INS-Cameroun, 2001), d'où la nécessité de prendre en compte l'amélioration de cet élevage dans la stratégie d'accroissement des productions animales.

Les poules de race locale sont à croissance lente, et malgré leurs effectifs 
élevés, elles contribuent seulement pour $10 \%$ du PIB et fournissent 20 œufs et $50 \%$ des $3600 \mathrm{~g}$ de viandes consommées par an par habitant au Cameroun (Ngou Ngoupayou, 1990; Fotsa et Manjeli, 2001; Fotsa et al., 2007). Cette faible contribution est imputable à la fois au système d'élevage, au faible potentiel génétique des animaux et à un manque d'investissement scientifique et économique.

A notre connaissance, très peu d'études ont été faites sur l'amélioration du potentiel génétique de la poule de race locale, principal matériel génétique de l'aviculture extensive. En zones urbaines et périurbaines, cette race est menacée d'extinction car elle est l'objet de métissage avec les races exotiques. Depuis plusieurs décennies, on assiste à un développement des élevages semi-intensifs et industriels des volailles (poules) autour des grands centres urbains et périurbains. Cette forme d'élevage constitue à n'en point douter un réservoir d'animaux de réforme de souche exotique qui, très souvent en divagation dans les villes contribuent à une dilution génétique des poules de race locale existante. Ce phénomène est moins accentué dans les zones rurales reculées. Les métissages entre la poule de race exotique et son homologue de race locale en zones urbaines et périurbaines pourraient justifier les observations de Keambou et al. (2007) qui trouvent qu'en général les poules de race locale des zones urbaines présentent des poids vifs adultes supérieurs à ceux de leurs compères des zones rurales. Toutefois, aucune investigation n'a encore vérifié ces assertions. L'un des préalables à l'amélioration génétique de la poule locale est une meilleure connaissance des caractéristiques zootechniques du matériel génétique dans son milieu d'élevage. Par ailleurs, l'évolution de la poule locale en milieu réel l'expose à un grand nombre de facteurs qui influencent l'expression des gènes sur le phénotype, et relativisent de ce fait les résultats obtenus en expérimentation classique (Hako et al., 2009). C'est pourquoi notre étude se propose d'apporter une contribution à la connaissance de la poule locale de la région de l'Ouest-Cameroun et plus spécifiquement, de comparer la qualité des œufs des poules et d'évaluer les performances de croissance des poussins des zones urbaines et rurales.

\section{MATERIEL ET METHODES \\ Site expérimental}

L'étude s'est déroulée dans la région des Hautes Terres de l'Ouest-Cameroun $\left(5^{\circ}\right.$ $7^{\circ} \mathrm{LN}$ et $8^{\circ}-20^{\circ}$ LE) (Figure 1$)$. Le climat de la zone d'étude est du type soudano-guinéen d'altitude. La température y varie de 16 à 27 ${ }^{\circ} \mathrm{C}$ et l'humidité relative oscille entre $40 \%$ durant les mois les plus secs (janvier-février) et $100 \%$ pour les mois les plus humides (juillet-août). La pluviométrie, $2000 \mathrm{~mm}$ par an en moyenne, est repartie sur une seule saison allant de mars à novembre (Hako et al., 2009).

\section{Cufs}

Les œufs utilisés dans cet essai ont été achetés dans trois localités urbaines (Dschang, Bafoussam, Mbouda) et trois localités rurales (Fokoué, Batcham et Bangang) de l'OuestCameroun. Les œufs collectés dans chaque zone ont été nettoyés et identifiés par lot. Un total de 320 œufs pour la zone rurale et 320 œufs pour la zone urbaine a donc été couvé dans différents compartiments d'un incubateur électrique de marque DETROIT ayant une capacité de 400 œufs. Le protocole d'incubation a été adapté de celui décrit par Esminger (1980): les œufs à incuber étaient ramassés 2 fois par jour, immédiatement nettoyés puis stockés à température ambiante, la chambre à air orientée vers le haut, pendant 10 jours avant l'incubation. Les œufs étaient ramenés dans la salle d'incubation $24 \mathrm{~h}$ avant la date de mise en incubateur. L'incubateur était nettoyé, désinfecté à l'eau javellisée et soumis à un vide sanitaire de deux jours avant l'introduction des œufs. La température $(37,5$ ${ }^{\circ} \mathrm{C}$ en moyenne) et l'hygrométrie (60-70\%) étaient vérifiées trois fois par jour lors de chacun des trois retournements journaliers des œufs, et si nécessaire corrigées. Le mirage se faisait au $7^{\text {ème }}$ et au $14^{\text {ème }}$ jour, et les œufs non fertiles éliminés à l'issu du dernier mirage. A partir du $18^{\text {ème }}$ jour les œufs n'étaient plus retournés jusqu'à l'éclosion.

\section{Conduite des animaux}

Dès l'éclosion, les poussins ont été pesés et transférés à la poussinière sur litière profonde de copeau de bois. Pendant toute la période de l'essai, les animaux avaient accès libre à l'eau et à l'aliment qui étaient disponibles en permanence, contenant 


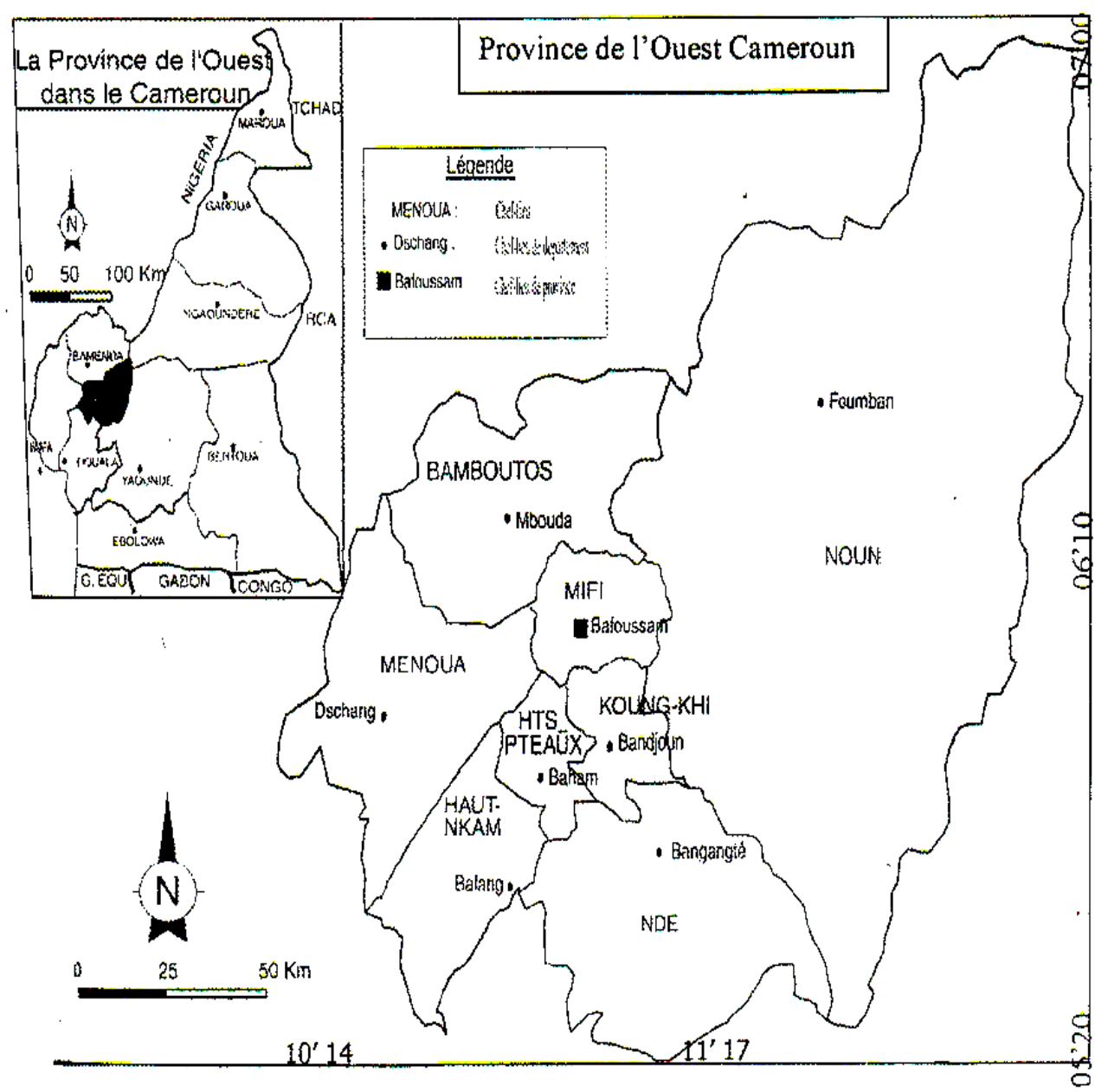

Figure 1: Carte de la situation géographique de l'Ouest-Cameroun (Source: Keambou, 2006).

$21,01 \%$ PB et 2909,06 kcal EM au démarrage et $18,70 \% \mathrm{~PB}$ et $3010,17 \mathrm{kcal} \mathrm{EM}$ à la finition. Les caractéristiques bromatologiques des aliments distribués aux poules sont présentées au tableau 1 .

\section{Collecte des données}

La couleur et la forme des œufs ont été décrites par observation visuelle. La longueur et la largeur des œufs ont été mesurées à l'aide d'un pied à coulisse (portée: $25 \mathrm{~cm}$ et de précision $0,02 \mathrm{~mm}$ ) et une balance (de marque Dial-0-Gram, OHAUS, de portée 610 $\mathrm{g}$ ayant une précision de $0,1 \mathrm{~g}$ ) a servi à la prise du poids des œufs.

A intervalle de sept jours, les poussins étaient pesés avec la même balance que les œufs. De même, les quantités d'aliment servies au cours de la semaine et les refus en fin de semaine ont été évalués par différence entre la quantité d'aliment distribuée dans la semaine et les résidus non consommés à la fin de la semaine avec la même balance sus mentionnée.

Les mensurations de l'œuf et le nombre d'œufs incubés, fertiles et éclos ont permis de calculer l'indice de l'œuf, le taux de fertilité et d'éclosion selon les formules de Esminger (1980), Il en a été de même pour les paramètres collectés pour le suivi de la croissance tels que le gain moyen quotidien (GMQ), la consommation moyenne hebdomadaire (CMH) par poussin et l'indice de consommation hebdomadaire (ICH) selon les formules suivantes :

Indice de l'œuf $=$ (Longueur / Largeur $) \times 100$ 
Taux de fertilité $=$ (Nombre œufs fécondés / Nombre œufs incubés) x 100

Taux d'éclosion $=$ (Nombre d'œufs éclos / Nombre d'œufs fécondés) x 100

Le gain moyen quotidien $(\mathrm{GMQ})=\sum$ (Poids en fin de semaine - poids en début de semaine) / $7 \mathrm{x}$ nombre de poussins

La consommation moyenne hebdomadaire par poussin $(\mathrm{CMH})=$ (quantité d'aliment servi au cours de la semaine - refus en fin de semaine) / nombre de poussins

L'indice de consommation hebdomadaire $\mathrm{ICH}=\mathrm{CMH} / 7 * \mathrm{GMQ}$

\section{Analyses statistiques}

Les données ont été analysées à l'aide de la statistique descriptive. Le test de Student a été utilisé pour la comparaison des mensurations moyennes au seuil de 5\%.

\section{RESULTATS}

Qualité des oufs de la poule locale des zones urbaines et rurales de l'OuestCameroun

\section{Forme et couleur de la coquille}

La forme et la couleur de la coquille des œufs de la poule locale des zones urbaines et rurales de l'Ouest-Cameroun sont présentées dans le tableau 2. Quelle que soit la zone, les œufs ont majoritairement la forme ovale. Toutefois, la proportion d'œufs de forme allongée en zone rurale $(13,1 \%)$ est plus de deux fois supérieure à celle de la zone urbaine $(05,0 \%)$. Indépendamment de la zone, la couleur de la coquille est soit blanche, brun foncé ou brun clair. Cependant, à zone égale, la couleur brune de la coquille est largement dominante.

\section{Poids, longueur et largeur}

Les résultats obtenus sont consignés dans le tableau 3. Il n'existe pas de différences significatives ( $p>0,05)$ entre les zones pour les caractères considérés, bien que les œufs issus des poules de la zone urbaine aient des mensurations supérieures à celles des œufs des poules de la zone rurale. Sur la base de ces performances, l'indice de l'œuf est estimé à environ 73 , inférieur à la norme requise de 75 pour les œufs devant être conditionnés dans les emballages standardisés (Smith, 1992).

\section{Taux de fertilité et d'éclosion}

Au tableau 4, on observe qu'entre les deux zones, les taux de fertilité sont significativement différents $(p \leq 0,05)$, contrairement aux taux d'éclosion qui ne diffèrent pas $(p>0,05)$. Dans les deux cas, les taux sont faibles et très inférieurs à ceux signalés dans la littérature. En effet, Nureldin (1989) rapporte des taux allant de 89,4 à 94,45\%. Cependant, Amayene (1991) a obtenu des taux de 67 et $49 \%$ en zones rurales et urbaines respectivement, assez proches de ceux de notre essai.

Tableau 1: Composition des rations alimentaires distribuées aux poules en démarrage et finition.

\begin{tabular}{lcc}
\hline \multirow{2}{*}{ Ingrédients } & \multicolumn{2}{c}{ Rations expérimentales } \\
\cline { 2 - 3 } & Démarrage & Finition \\
\hline Maïs & 54 & 60 \\
Remoulage & 9 & 10 \\
Farine de soja & 20 & 11 \\
Farine de coton & 2 & 10 \\
Farine de poisson & 6 & 6 \\
Huile de palme & 2 & 1 \\
Farine de coquillages & 1,8 & 1,5 \\
Farine d'os & 0,8 & 3 \\
Sel & 0,3 & 0,5 \\
CAMV (5\%) & 5 & 1 \\
\hline Total & $\mathbf{1 0 0}$ & $\mathbf{1 0 0}$ \\
\hline Energie métabolisable & 2909,06 & 3010,17 \\
(EM/kg) & 21,01 & 18,70 \\
Protéines brutes (\%) & 1,16 & 0,81 \\
Lysine & 0,41 & 0,33 \\
Méthionine & 1,17 & 0,90 \\
Calcium & 0,42 & 0,36 \\
Phosphore & 3,90 & 3,70 \\
Cellulose brute & Amino-Vitaminique \\
\hline CAMV (5\%) $=$ & Complexe \\
(concentré 5\%) & \multicolumn{2}{|}{}
\end{tabular}

Tableau 2: Forme et couleur de la coquille des œufs de la poule locale des zones urbaines et rurales de l'Ouest-Cameroun.

\begin{tabular}{lcccc}
\hline \multirow{2}{*}{ Caractères } & \multicolumn{2}{c}{ Zone rurale } & \multicolumn{2}{c}{ Zone urbaine } \\
\cline { 2 - 5 } & $\mathrm{n}$ & $\%$ & $\mathrm{n}$ & $\%$ \\
\hline Forme & & & & \\
Ovale & 278 & 86,9 & 304 & 95,0 \\
Allongée & 42 & 13,1 & 16 & 05,0 \\
$\quad$ Total & $\mathbf{3 2 0}$ & $\mathbf{1 0 0}$ & $\mathbf{3 2 0}$ & $\mathbf{1 0 0 , 0 0}$ \\
Couleur de la coquille & & & \\
$\quad$ Blanc & 44 & 13,75 & 53 & 16,6 \\
Brun clair & 139 & 43,44 & 110 & 34,4 \\
Brun foncé & 137 & 42,81 & 157 & 49,0 \\
Total & $\mathbf{3 2 0}$ & $\mathbf{1 0 0 , 0 0}$ & $\mathbf{3 2 0}$ & $\mathbf{1 0 0 , 0 0}$ \\
\hline n= nombre d'œufs, \%= pourcentage du total.
\end{tabular}


Tableau 3: Poids et mensurations des œufs de la poule locale des zones urbaines et rurales de l'Ouest-Cameroun.

\begin{tabular}{|c|c|c|c|c|}
\hline \multirow{2}{*}{ Caractères } & \multicolumn{2}{|c|}{ Zone rurale } & \multicolumn{2}{|c|}{ Zone urbaine } \\
\hline & $\mathrm{n}$ & Moy. \pm e.t & $\mathrm{n}$ & Moy.土 e.t \\
\hline Poids (g) & 320 & $44,89 \pm 04,99^{a}$ & 320 & $45,94 \pm 05,73^{a}$ \\
\hline Longueur (mm) & 320 & $54,26 \pm 02,67^{\mathrm{a}}$ & 320 & $54,32 \pm 02,62^{\mathrm{a}}$ \\
\hline Largeur (mm) & 320 & $39,43 \pm 01,57^{\mathrm{a}}$ & 320 & $39,68 \pm 02,32^{\mathrm{a}}$ \\
\hline Indice de l'œuf & 320 & $72,67^{\mathrm{a}}$ & 320 & $73,04^{\mathrm{a}}$ \\
\hline
\end{tabular}

Tableau 4: Nombre d'œufs incubés, taux de fertilité et d'éclosion de la poule locale des zones rurales et urbaines de l'Ouest-Cameroun.

\begin{tabular}{lcc}
\hline Paramètres & Zone rurale & Zone urbaine \\
\hline Nombre d'œufs incubés & 310 & 308 \\
Taux de fertilité au 14 $4^{\text {ème }}$ jour $(\%)$ & $64,2^{\mathrm{a}}$ & $43,5^{\mathrm{b}}$ \\
Taux d'éclosion (\%) & $62,3^{\mathrm{a}}$ & $59,7^{\mathrm{a}}$ \\
\hline
\end{tabular}

a, b. sur la même ligne, les valeurs affectées de la même lettre ne sont pas significativement différentes $(\mathrm{p}>0,05)$.

\section{Performances de croissance de la poule locale des zones urbaines et rurales de l'Ouest-Cameroun}

D'après le tableau 5 , il n'y a pas de différences significatives pour tous les paramètres étudiés entre les deux zones de chaque âge. Toutefois, de l'éclosion jusqu'à la $5^{\text {ième }}$ semaine, les poussins de la zone urbaine sont plus lourds que ceux de la zone rurale bien que les différences de poids ne soient pas significatives $(p>0,05)$. Les poids des poussins obtenus à cinq semaines d'âge dans cet essai sont largement supérieurs à ceux présentés par Tchoumboué et al. (2000) chez les poules en divagation $(198.5 \mathrm{~g})$ et les poids à l'éclosion assez proches des résultats de Hassen et al. (2006) (27,8 g). La consommation hebdomadaire et l'indice de consommation présentent une évolution en dents de scie. Le gain moyen quotidien, tout au long de la durée de l'essai est plus faible chez le lot des zones rurales exception faite de la $4^{\text {ème }}$ semaine. Les indices de consommation à la $4^{\text {ième }}$ et $5^{\text {ième }}$ semaine ont été supérieurs à ceux rapportés par Roy et al. (2004) $(3,10$ et 3,26$)$ alors que les consommations hebdomadaires des poussins durant les mêmes périodes ont été inférieurs aux résultats obtenus par Hassen et al. (2006) (537,6 et 516).

De la première à la quatrième semaine, la consommation alimentaire est supérieure chez les poussins de la zone rurale, et devient inférieure à celle des poussins de la zone urbaine après la quatrième semaine. La forte consommation des poussins de la zone rurale dans les quatre premières semaines serait due à leur intense activité durant cette période, contrairement à ceux de la zone urbaine. Chez ces derniers la consommation devient plus importante après la quatrième semaine, telle qu'observée avec les souches exotiques. Ceci pourrait de nouveau suggérer un possible métissage en zone urbaine.

L'indice de consommation chez les poussins de la zone rurale est strictement croissant avec l'âge des animaux, alors que celui des poussins de la zone urbaine présente une inflexion entre la première et la troisième semaine.

En général, le gain moyen quotidien a été plus élevé chez les poussins de la zone urbaine, sauf à la quatrième semaine. 
Tableau 5: Evolution du poids (g), de la consommation alimentaire (g) de l'indice de consommation et du gain moyen quotidien (g) de la poule locale des zones urbaines et rurales de l'Ouest-Cameroun en fonction de l'âge.

\begin{tabular}{llccccc}
\hline $\begin{array}{l}\text { Age } \\
\text { (semaine) }\end{array}$ & Zones & $\mathbf{n}$ & $\begin{array}{c}\text { Poids } \\
(\mathbf{g})\end{array}$ & $\begin{array}{c}\text { Consommation } \\
(\mathbf{g})\end{array}$ & $\begin{array}{c}\text { Indice de } \\
\text { consommation }\end{array}$ & $\begin{array}{c}\text { Gain moyen } \\
\text { quotidien }(\mathbf{g})\end{array}$ \\
\hline 0 & Rurale & 124 & $28,72 \pm 03,86$ & & & \\
(éclosion) & Urbaine & 80 & $30,41 \pm 03,63$ & & & \\
\multirow{2}{*}{1} & Rurale & 124 & $52,31 \pm 10,28$ & 50,54 & 2,14 & \\
& Urbaine & 80 & $54,21 \pm 08,54$ & 49,59 & 2,92 & \\
2 & Rurale & 124 & $87,70 \pm 18,42$ & 94,83 & 2,67 & $5,06 \pm 1,58$ \\
& Urbaine & 80 & $91,47 \pm 17,75$ & 71,42 & 1,91 & $5,32 \pm 1,45$ \\
3 & Rurale & 122 & $120,88 \pm 32,19$ & 150,96 & 4,49 & $4,80 \pm 2,88$ \\
& Urbaine & 80 & $145,93 \pm 33,98$ & 155,60 & 2,84 & $7,80 \pm 2,60$ \\
4 & Rurale & 122 & $192,65 \pm 55,49$ & 333,30 & 4,64 & $10,25 \pm 04,60$ \\
& Urbaine & 80 & $200,45 \pm 54,49$ & 310,71 & 5,70 & $7,78 \pm 3,34$ \\
5 & Rurale & 122 & $254,72 \pm 77,92$ & 366,60 & 6,07 & $8,61 \pm 4,40$ \\
& Urbaine & 80 & $273,30 \pm 74,64$ & 428,57 & 5,83 & $10,41 \pm 03,31$ \\
\hline \multirow{2}{*}{ Moyenne } & Rurale & & & $\mathbf{1 9 9 , 2 5}$ & $\mathbf{4 , 0 0}$ & $\mathbf{7 , 1 8 \pm 3 , 3 6}$ \\
& Urbaine & & & $\mathbf{2 0 3 , 1 8}$ & $\mathbf{3 , 8 4}$ & $\mathbf{7 , 8 2 \pm 2 , 6 7}$ \\
\hline
\end{tabular}

$\mathrm{n}=$ nombre de poussins

\section{DISCUSSION}

La forme des oufs est un caractère héréditaire. Il est montré que les œufs sélectionnés pour l'incubation doivent être de forme ovale (Austic, et Nesheim, 1990). La prédominance de la forme ovale d'œufs démontre que la poule locale est une bonne reproductrice. Par contre, les œufs allongés présentent le risque de se casser lorsqu'on les range dans des plateaux alvéolés standard.

$$
\text { Les résultats montrant une }
$$

prédominance d'œufs à coquille brun clair et brun foncé ne concordent pas avec ceux de Missohou et al. (1998) qui a observé chez la poule locale du Sénégal une forte majorité d'œufs à coquilles blanches et seulement $27 \%$ d'œufs à coquilles rousses. La couleur de la coquille étant influencée par la race de la poule, cette différence de coloration pourrait signifier une variabilité génétique entre les poules de Sénégal et celles de l'OuestCameroun. Cette différence pourrait être liée à une sélection naturelle ou proviendrait de l'effet des métissages entre la poule locale de l'Ouest-Cameroun et les souches exotiques à coquille brune. La couleur de la coquille est importante dans de nombreux pays et spécifiquement au Royaume Uni où les consommateurs préfèrent les œufs à coquilles brunes aux œufs à coquilles blanches et payent d'ailleurs ceux-ci plus cher. Pour cette raison et bien que les races productrices d'œufs à coquilles brunes soient moins productives que celles produisant les œufs à coquilles blanches, les éleveurs ont ces dix dernières années fournis des efforts concertés afin de produire des souches hautement productives de pondeuses d'œufs à coquilles brunes. Cette tâche s'est avérée doublement compliquée du fait que plus l'oiseau produit, plus les coquilles deviennent blanches (Smith, 1992).

Les écarts observés entre les taux de fertilité des oufs seraient imputables aux différences génétiques ainsi qu'aux conditions d'élevage (activité sexuelle réduite chez les mâles, éventuelles pathologies, mauvaise alimentation, utilisation progressive des organophosphorés dans la protection phytosanitaire). 
Les taux d'éclosion dans les deux zones restent en dessous des $80-90 \%$ rapportés par Jourdain (1980). Ceci pourrait être une qualité propre des œufs ou attribuable aux conditions d'incubation. En effet, de fréquentes coupures d'électricité sont intervenues pendant la période de l'essai, ces dernières lorsqu'elles sont prolongées ou très répétitives sont susceptibles de provoquer des mortalités embryonnaires, et donc influencer l'éclosabilité des œufs.

Les métissages pourraient justifier la légère supériorité, bien que non significative, du poids des œufs issus des animaux de la zone urbaine. Toutefois, le poids des œufs de notre échantillon dans les deux zones est supérieur aux 30,7 et $37,5 \mathrm{~g}$ obtenus respectivement par Mourad et al. (1997) et Missohou et al. (1998), mais assez proches des 41 et $44 \mathrm{~g}$ estimés respectivement par Minga et al. (1989) et Tchoumboué et al. (2000). Les différences génétiques pourraient expliquer ces divergences de poids. L'action $\mathrm{du}$ milieu et particulièrement les conditions d'élevage légèrement améliorées en milieu urbain, ne devrait pas non plus être négligée. Par ailleurs, la ressource alimentaire résiduelle picorable est influencée par le climat local et les saisons (Olukosi et Sonaiya, 2003 ; Rashid et al., 2005), ce qui en retour affecte le poids des animaux et de leurs œufs. Ceci pourrait également expliquer la variation du poids des œufs issus des zones semi-arides obtenus par Mourad et al. (1997) et Missohou et al. (1998) respectivement en Guinée et au Sénégal, par rapport à celui des œufs des zones plus humides tel que obtenus par Minga et al. (1989) et Tchoumboué et al. (2000) respectivement en Tanzanie et au Cameroun. La longueur et la largeur des oeufs obtenues dans notre essai sont supérieures aux valeurs de 35,24 et 23,59 mm présentées par Fayeye et al. (2005); ceci pourrait également être dû aux différences génétiques. Pour toutes ces raisons, on pourrait dire que la poule locale de l'Ouest-Cameroun présente des œufs beaucoup plus gros que ses homologues des autres pays africains rencontrés dans la littérature.

Les performances supérieures de nos poussins, élevés dans des conditions améliorées justifient l'hypothèse selon laquelle les conditions d'élevage peuvent dans une certaine mesure être facteur d'amélioration de la productivité de la poule locale (Tchoumboué et al., 2000). La supériorité du poids et des gains moyens quotidiens des poussins de la zone urbaine est attribuable soit aux conditions d'élevage des parentaux probablement meilleures; soit à d'éventuels métissages de la poule locale avec les souches exotiques. La variabilité de consommation alimentaire et indice de consommation entre les écotypes seraient probablement le fait du gaspillage observé après la $3^{\text {ième }}$ semaine, particulièrement par les poussins des zones rurales instinctivement plus actifs, et en dépit des mangeoires siphoïdes utilisées. Les différences obtenues par rapport aux autres auteurs seraient probablement liées aux types génétiques des différents milieux d'études.

\section{Conclusion}

$\mathrm{Au}$ terme de cette étude qui avait pour objectifs la comparaison de la qualité des œufs des poules et l'évaluation des performances de croissance des poussins locaux des zones urbaines et rurales de l'Ouest-Cameroun, on peut noter que :

- Les œufs de poules locales présentent des caractéristiques morphologiques et éclosabilités comparables entre les zones urbaines et rurales de l'Ouest-Cameroun,

- Le taux de fertilité est plus élevé en zone rurale qu'en zone urbaine,

- Le poids vif de l'éclosion à la $5^{\text {ième }}$ semaine d'élevage est sensiblement plus élevé chez les poussins de la zone urbaine mais sans différence significative,

- La consommation est plus élevée chez les poussins de la zone rurale durant les quatre premières semaines d'élevage et devient plus élevée chez les poussins de la zone urbaine après cette période.

L'évolution de l'indice de consommation et du gain moyen quotidien montre que la production de poussins locaux serait économiquement plus rentable au cours des quatre premières semaines. La supériorité apparente et la variabilité des performances obtenues au niveau des mensurations des œufs et des performances de croissance, comparativement aux résultats obtenus dans d'autres pays africains, peut bien attester qu'une sélection fructueuse peut être conduite sur la base de ces caractères au sein de la population de poules locales de l'Ouest- 
Cameroun. Il serait souhaitable que l'étude soit étendue à d'autres zones agroécologiques, sur une durée plus longue et un cheptel plus grand pour apporter plus de précision aux résultats et éventuellement au choix de la méthode d'amélioration génétique de la poule locale.

\section{REFERENCES BIBLIOGRAPHIQUES}

Amayene P. 1991. Comparaison de quelques paramètres de reproduction et de croissance des poulets locaux du Cameroun à ceux de la Rhode Island Red. Mémoire de fin de cycle. INADER, Dschang, $62 \mathrm{p}$.

Austic RE, Nesheim MC. 1990. Poultry Production $\left(13^{\text {th }}\right.$ edn). Lippincott Williams and Wilkins; 325p.

Esminger ME. 1980. Poultry Science (Animal Agriculture Series) (2nd edn). The Interstate Printers and Publisher Inc.; $502 \mathrm{p}$.

Fayeye TR, Adeshiyan AB, Olugbami AA. 2005. Eggs traits, hatchability and early growth performance of Fulani-ecotype chicken. Livestock Research for Rural Development, 17(8): 2005.

Fotsa JC, Manjeli Y. 2001. Analyse comparée des performances de croissance en claustration des poussins de souche locale d'une lignée Jupiter et de leurs croisements $\mathrm{F}_{1}$. Annales des Sciences Agronomiques du Bénin, 2: 181-192.

Fotsa JC, Rognon X, Tixier-Boichard M, Ngou Ngoupayou JD, Poné Kamdem D, Manjeli Y, Bordas A. 2007. Exploitation de la poule locale (Gallus gallus) en zone de forêt humide du Cameroun. Bull. Anim. Hlth. Prod. Afr., 55:59-73.

Hako Touko BA, Manjeli Y, Teguia A, Tchoumboué J. 2009. Evaluation et prédiction de l'effet du type génétique sur l'évolution du poids vif de la poule locale camerounaise (Gallus domesticus). Livestock Research for Rural Development. Volume 21, Article \#31. Retrieved March 11, 2009, from http://www.lrrd.org/lrrd21/3/hako21031.h tm.

Hassen H, Neser FWC, Dessie T, De Rock A, Van Marle-Koster E. 2006. Studies on the growth performance of native chicken ecotypes and RIR chichen under improved management system in
Northwest Ethiopia. Livestoc Research for Rural Development, 18(6): 2006.

INS-Cameroun. 2001. Institut National des Statistiques: Note annuelle sur le secteur primaire : Agriculture, élevage, pêche, 30.

Jourdain R. 1980. L'Aviculture en Milieu Tropical. Edition Sté Jourdain, Boissy le Chatel: France; $148 \mathrm{p}$.

Keambou TC. 2006. Caractères morphologiques, mensurations corporel-les et diversité phylogénétique de la poule locale hautes terres de l'Ouest Cameroun. Thèse de Master of Science, Faculté d'Agronomie et des Sciences Agricoles, Université de Dschang-Cameroun, 71p.

Keambou TC, Manjeli Y, Tchoumboué J, Teguia A, Iroume RN. 2007. Caractérisation morphobiométrique des ressources génétiques de poules locales des hautes terres de l'ouest Cameroun. Livestock Research for Rural Development. Volume 19, Article \#107. Retrieved August 10, 2007, from http://www.cipav.org.co/lrrd/lrrd19/8/kea m19107.htm.

Missohou A, Sow RS, Ngwe Assoumou C. 1998. Caractéristiques morphobiométriques de la poule du Sénégal, Animal Genetic Resource Information, 24: 63-69.

Minga UM, Katule A, Maeda T, Musasa J. 1989. Potential and problems of the traditional chicken industry in Tanzania. Proceedings of the $7^{\text {th }}$ Tanzania Veterinary Association Scientific Conference; 207-215.

Mourad M, Bah AS, Gbanamou. 1997. Evaluation de la productivité et de la mortalité de la poule locale sur le plateau du Sankara, Faranah, Guinée en 19931994. Revue Elevage et Médecine Vétérinaire des Pays Tropicaux, 50: 343349.

Ngou NJD. 1990. Country report on small holder rural poultry production in Cameroon. CTA Seminar Proceedings on Small Holder Rural Poultry Production. 9-13 October1990, Thessaloniki: Greece; 2: $39-41$

Nureldin M. 1989. Rural poultry production in Sudan. Proceedings of an International Workshop on Rural Poultry Development in Africa, Sonaiya EB (ed). ILE-IFE: Nigeria; 160-165. 
Olukosi OA, Sonaiya EB. 2003. Determination of the quantity of scavengeable feed for family poultry on free range. Livestock Research for Rural Development, 15(5): 2003.

Rashid MM, Islam MN, Roy BC, Jacobsen K, Lauridsen C. 2005. Nutrient concentration of crop and gizzard content of indigenous scavenging chickens under rural conditions of Bangladesh. Livestock Research for Rural Development, 17(2): 2005.

Roy BC, Ranvig H, Chowdhury SD, Rashid MM, Faruque MR. 2004. Production of day-old chicks from crossbred chicken eggs by broody hens, rice husk incubator and electric incubator, and their rearing up to 6 weeks. Livestock Research for Rural Develoment, 16(3): 2004.

Smith AJ. 1992. L'Elevage de la Volaille. Le Technicien d'Agriculture Tropicale (Vol 1). Maisonneuve et Larose, CTA; 183p.

Tchoumboué J, Manjeli Y, Téguia A, Ewane NJ. 2000. Productivité et effets comparés de trois systèmes de conduite de l'élevage sur les performances de l'aviculture villageoise dans les hautes terres de l'Ouest-Cameroun. Science Agronomique et Développement, 2(1): 6-14. 